\title{
Communication Equilibria with Partially Verifiable Types ${ }^{1}$
}

\author{
Françoise Forges $^{2}$ Frédéric Koessler ${ }^{3}$ \\ THEMA (CNRS, UMR 7536) \\ Université de Cergy-Pontoise \\ 33, Boulevard du Port \\ 95011 Cergy-Pontoise, France
}

May 20, 2003

\footnotetext{
${ }^{1}$ We thank Raymond Deneckere, Gabriel Desgranges, Jean-François Mertens, Regis Renault, Sergei Severinov and seminar participants at the Game Theory Seminar at Institut Henri Poincaré (Paris), the Max Planck Institute for Research into Economic Systems (Jena), Maison des Sciences Economiques (Paris), and the University of Paris X-Nanterre for helpful comments and discussions.

${ }^{2}$ E-mail: Francoise.Forges@eco.u-cergy.fr.

${ }^{3}$ E-mail: Frederic.Koessler@eco.u-cergy.fr.
} 


\begin{abstract}
This paper studies the set of equilibria that can be achieved by adding general communication systems to Bayesian games in which some information can be certified or, equivalently, in which players' types are partially verifiable. Certifiability of information is formalized by a set of available reports for each player that varies with the true state of the world. Given these state-dependent sets of reports, we characterize canonical equilibria for which generalized versions of the revelation principle are valid. Communication equilibria and associated canonical representations are obtained as special cases when no information can be certified.
\end{abstract}

Keywords: Bayesian game; Communication equilibrium; Information certification; Revelation principle; Verifiable types.

JEL Classification: C72; D82.

\title{
Résumé
}

Cet article étudie l'ensemble des équilibres que l'on peut atteindre en ajoutant des systèmes de communication généraux à des jeux bayésiens dans lesquels certaines informations peuvent être certifiées ou, de façon équivalente, dans lesquels les types des joueurs sont partiellement vérifiables. Le caractère certifiable de l'information est formalisé par le fait que chaque joueur dispose d'un ensemble de messages dépendant de l'état de la nature. Étant donné un tel ensemble de messages, nous caractérisons des équilibres canoniques auxquels s'appliquent des versions généralisées du principe de révélation. Les équilibres en communication et leurs représentations canoniques correspondent au cas particulier où aucune information ne peut être certifiée. 


\section{Introduction}

Since the pioneering work of Aumann (1974) on correlated equilibria and Crawford and Sobel's (1982) analysis of cheap talk games, the introduction of communication possibilities into the analysis of interactive decision situations has been commonplace in a whole host of applied and theoretical researches (for some recent references, see, e.g., Aumann and Hart, 2003, Baliga and Morris, 2002, Ben-Porath, 2003, Gerardi, 2003, Krishna and Morgan, 2002, and Urbano and Vila, 2002). Such analyses are motivated by the fact that when individuals can talk to each other before choosing their final payoff-relevant actions, they may be able to share information and/or agree on compromises, and then reach outcomes that differ from those of the standard Nash equilibrium solution concept. For example, a correlated equilibrium of a strategic form game is a Nash equilibrium of some extension of the game where players receive private, "extraneous" and possibly correlated signals before the beginning of the original game. Such a solution concept is appropriate to characterize the set of all equilibrium outcomes achievable in one-shot complete information games with costless and non-binding communication.

With the exception of some specific applications discussed below, the literature on communication games and the various extensions of the correlated equilibrium to incomplete information typically relied on the assumption that the set of reports available to a player does not depend on his private information. ${ }^{1}$ On the contrary, our starting point in this paper is to allow the set of all possible messages that an individual is able to send to vary with his actual state of knowledge. Said differently, the information that is transmitted might be certifiable or provable by its sender, or verifiable by its receiver. ${ }^{2}$ For example, reports may consist of written documents or direct physical observations which may not be forged. ${ }^{3}$ Alternatively, in economic or legal interactions there may be penalties for perjury, false advertising and warranty violations, or accounting principles that impose limits on what is possible to disclose. Requiring traders in an exchange economy to deposit collateral for each order (as, e.g., in Forges, Mertens, and Vohra, 2002) also implies that their types are partially verifiable because traders are not able to over-report their initial endowments. ${ }^{4}$ Finally, an individual's ability to manipulate and misrepresent information may be limited due to psychological reasons (e.g., observable emotions such as blushing, or a strong taste for honesty that cannot adequately be represented by standard preferences, as in Alger and Ma, 2003, and Alger and Renault, 2002). The purpose of this paper is precisely to study in a general and tractable framework the

\footnotetext{
${ }^{1}$ For an overview, see, e.g., Farrell and Rabin (1996), and Myerson (1994).

${ }^{2}$ In this paper, the terms "certifiable", "provable" and "verifiable" are equivalent. See subsection 3.1 for a formal definition.

${ }^{3}$ For instance, disclosures of knowledge generated by R\&D may be knowledge-dependent in the sense that an informed firm cannot disclose more knowledge than it has (see, e.g., d'Aspremont, Bhattacharya, and Gérard-Varet, 2000).

${ }^{4}$ Similarly, the type of a budget-constrained buyer may be partially verifiable if the seller can ask him to post a bond equal to his reported budget (as, e.g., in Che and Gale, 2000).
} 
effects of adding communication systems to incomplete information games in which players' types are partially verifiable, and to provide a canonical representation of the equilibria of such extended communication games.

Our basic model is an $n$-person Bayesian game. As in Forges (1990), we extend the game by allowing the players to communicate for several periods, with the help of a mediator, before they make their decisions. More precisely, at every stage of the extended game, every player sends an input to a communication device, which selects a private output for every player, as a function of past inputs and outputs. In a standard communication equilibrium, all types of a given player have access to the same inputs, which are thus interpreted as cheap talk. Here, we assume that, in addition to these messages, each player can also transmit reports from a type-dependent set, i.e., can send certified information into the communication system. We define a certification equilibrium as a Nash equilibrium of such an extension of the Bayesian game. Our first result (Theorem 1) is a characterization of all certification equilibrium outcomes that can be achieved for given sets of type-dependent reports for every player. We first show that the type-dependent report sets can be represented in a canonical way, in terms of the fundamentals of the game (we will refer to this representation as to a certifiability configuration). Once such a certifiability configuration is well-defined, the canonical representation we propose is simple: players are only required to present, in a one-stage game, the most informative certificate concerning their type to a mediator and to make a cheap talk claim about their type. Then, once the mediator has received a report in this canonical space from each player he makes private recommendations to the players. We show that there is no loss of generality in focusing on such representations and on equilibria where players reveal their true type and follow the recommendations of the mediator. This result can be interpreted as the generalized revelation principle for Bayesian games with partially verifiable types. The associated canonical representation (resp., canonical equilibrium) is the analog of a direct mechanism (resp., direct incentive-compatible mechanism) used in the mechanism design literature.

If the original set of possible communication systems is restrained to one-period communication systems where players can only present one verifiable argument, we also provide (in Theorem 2) a sufficient condition on the certifiability configuration which maintains the outcome equivalence between the associated certification equilibria and canonical certification equilibria. Finally, Theorem 3 is even closer to the traditional revelation principle than the previous results. It states that every certification equilibrium outcome can be achieved as a truthful and obedient equilibrium of a one-stage communication extension of the game in which the set of reports of every player is just a subset of his original set of types. In this scenario, it is implicitly assumed that players must produce a certificate that is consistent with the type they report. By contrast to Theorems 1 and 2, Theorem 3 does not describe a full equivalence. Its converse holds under further assumptions, which guarantee that the mediator can restrain the set of reports available in the communication system. 
Our approach combines three areas of research. As made clear above, the first relates to the notion of communication equilibrium (Forges, 1986; Myerson, 1982, 1986). The second area of research related to our work is the economic literature dealing with strategic information revelation, initiated by Grossman (1981), Grossman and Hart (1980) and Milgrom (1981), which investigates the amount of information voluntarily transmitted when individuals are required to make only truthfulbut possibly very vague - disclosures. ${ }^{5}$ This literature includes specific applications in oligopoly theory (see, e.g., Okuno-Fujiwara, Postlewaite, and Suzumura, 1990), finance (see, e.g., Shin, 2003), and law (see, e.g., Shin, 1994). The accounting literature has also placed considerable emphasis on games with strategic information revelation (see, e.g., Verrecchia, 2001 and references therein). Contrary to those previous contributions we consider a general game-theoretical framework allowing private, stochastic, repeated, and mediated information revelation, and we do not require players' types to be independent. Finally, our work is related to the literature on mechanism design with partially verifiable information (Bull and Watson, 2002, Deneckere and Severinov, 2001, Green and Laffont, 1986). This literature, which is restricted to the implementation of an exogenous social choice function, studies the validity of the standard revelation principle when the set of available reports of a single informed agent (or several symmetrically informed agents) varies with the true state of the world.

Green and Laffont (1986) pointed out that the revelation principle might fail in this framework, and proposed the 'nested range condition' as a necessary and sufficient condition on the report sets for a form of the revelation principle to hold. It was implicit in their approach that the agent could only send a single message, typically consisting of a type. Deneckere and Severinov (2001) showed that the revelation principle could be restored by enlarging the agent's set of possible reports. Both papers focus on message spaces that are closely related to the original state spaces, i.e., on direct mechanisms so that the point of the revelation principle is truthful implementation. The difference between this paper and those contributions is that our revelation principle applies to $n$-person games, in which the players have asymmetric information and must make decisions. Furthermore, instead of starting with some desirable outcome function and looking for the means to implement it as an equilibrium outcome, we are rather interested in characterizing all (possibly mixed) equilibrium outcomes that are feasible when general means of communication (i.e., mediators with perfect recall, equipped with lotteries, for several periods) are available to the players. In particular, the sets of (type-dependent as well as typeindependent) inputs and the sets of outputs of non-canonical communication systems are fully arbitrary, their elements have no pre-determined semantic meaning. This is the reason why we insist in establishing full equivalence results, stating not only that all equilibrium outcomes can be achieved as canonical ones but also that the set of

\footnotetext{
${ }^{5}$ For more recent references, see, e.g., Glazer and Rubinstein (2001), Koessler (2002, 2003), Lipman and Seppi (1995), Seidmann and Winter (1997), and Wolinsky (2003).
} 
canonical outcomes is not too large, i.e., that all canonical outcomes are compatible with the original certification possibilities. In this way, our representations can be used without loss of generality to maximize any function of the players' payoffs.

The paper is organized as follows. In Section 2 we present our general framework and some preliminary definitions. Canonical representations and generalized versions of the revelation principle for Bayesian games are analyzed in Section 3. We conclude in Section 4. The Appendix contains the proofs.

\section{General Framework and Definitions}

\subsection{Bayesian Games and Communication Systems}

We represent an interactive decision situation under asymmetric information by a (finite) Bayesian game

$$
G=\left\langle N,\left(A_{i}\right)_{i \in N},\left(T_{i}\right)_{i \in N}, p,\left(u_{i}\right)_{i \in N}\right\rangle
$$

where $N=\{1, \ldots, n\}$ is the set of players, $A_{i}$ is player $i$ 's set of possible actions, $T_{i}$ is player $i$ 's set of possible types, $p \in \Delta(T)$ is a common prior probability distribution over the set of type profiles $T=\prod_{i \in N} T_{i}$, and $u_{i}: A \times T \rightarrow \mathbb{R}$ is player $i$ 's state dependent payoff (utility) function, where $A=\prod_{i \in N} A_{i}$ is the set of action profiles. Let $p\left(t_{i}\right) \equiv \sum_{t_{-i} \in T_{-i}} p\left(t_{i}, t_{-i}\right)$ be the prior probability that player $i$ 's type is $t_{i}{ }^{6}$ We assume without loss of generality that $p\left(t_{i}\right)>0$ for all $i \in N$ and $t_{i} \in T_{i}$. Let $p\left(t_{-i} \mid t_{i}\right) \equiv \frac{p(t)}{p\left(t_{i}\right)}$ be the subjective probability that player $i$ assigns to the event that $t_{-i}$ is the actual profile of the other players' types if his own type is $t_{i} \cdot{ }^{7}$

To allow players to communicate before choosing an action in the Bayesian game $G$, we introduce a communication system (or mediator) that helps players to share information and to coordinate their actions. ${ }^{8}$ As usual, in a game with communication players exchange messages conditionally on past messages and on their own type before choosing their actions. However, contrary to previous work related to cheap talk communication and to the various extensions of the correlated equilibrium to incomplete information, we assume that the set of available messages may be type-dependent. As a consequence, reports may have some pure informational content which does not depend on any particular equilibrium and players may be able to certify some of their information.

Formally, a (finite) communication system given the set of players, $N$, and the set of possible type profiles, $T$, is denoted by

$$
c=\left\langle\left(R_{i}\right)_{i \in N},\left(S_{i}\right)_{i \in N},\left(M_{i}\right)_{i \in N}, K,\left(\nu^{k}\right)_{k=0,1, \ldots, K}\right\rangle .
$$

\footnotetext{
${ }^{6}$ For any variable, we denote its profile over all agents except that of player $i$ by the corresponding letter with subscript $-i$.

${ }^{7}$ We do not assume that every type profile has non-zero probability.

${ }^{8}$ Players have no ability to sign any contract or binding agreement. Hence, our approach is strictly non-cooperative.
} 
The positive integer $K$ is the number of communication periods. For each player $i$, $R_{i}: T_{i} \rightarrow \mathcal{R}_{i}$ is a reporting correspondence that determines the set $R_{i}\left(t_{i}\right)$ of typedependent inputs available to player $i$ of type $t_{i} \in T_{i}$, i.e., the set of reports that player $i$ can send out into the communication system in each period if his actual type is $t_{i}$, and $\mathcal{R}_{i} \equiv \bigcup_{t_{i} \in T_{i}} R_{i}\left(t_{i}\right)$ is the set of all reports the communication system can receive from player $i$ in each period. The set $S_{i}$ is the set of type-independent inputs available to player $i$, i.e., the set of cheap talk signals that player $i$ can send out into the communication system in each period. The set $M_{i}$ is the set of outputs for player $i$, i.e., the set of all messages that player $i$ can privately receive from the communication system in each period. Let $\mathcal{R}=\prod_{i \in N} \mathcal{R}_{i}, S=\prod_{i \in N} S_{i}$, and $M=\prod_{i \in N} M_{i}$. (Observe that, a priori, the elements of $\mathcal{R}, S$ and $M$ have no semantic content.) In period 0 , each player $i$ privately receives from the communication system an initial output $m_{i}^{0} \in M_{i}$, where $m^{0}=\left(m_{i}^{0}\right)_{i \in N}$ is distributed according to the probability distribution $\nu^{0} \in \Delta(M)$. Then, at the end of each communication period $k \in\{1, \ldots, K\}$, after all inputs up to that period have been received by the communication system, the transition probability

$$
\nu^{k}: M^{k} \times \mathcal{R}^{k} \times S^{k} \rightarrow \Delta(M),
$$

chooses the outputs as a function of past outputs and past and present inputs. That is, $\nu\left(m^{k} \mid m^{0}, m^{1}, \ldots, m^{k-1}, r^{1}, \ldots, r^{k}, s^{1}, \ldots, s^{k}\right)$ is the conditional probability that $m^{k}=\left(m_{1}^{k}, \ldots, m_{n}^{k}\right) \in M$ are the messages privately received by the various players at the end of period $k$ given the sequence of vectors of past outputs $\left(m^{0}, m^{1}, \ldots, m^{k-1}\right) \in M^{k}$, past and present type-dependent inputs $\left(r^{1}, \ldots, r^{k}\right) \in$ $\mathcal{R}^{k}$, and past and present type-independent inputs $\left(s^{1}, \ldots, s^{k}\right) \in S^{k}$.

\subsection{Extended Bayesian Games and Certification Equilibria}

Given a communication system $c$, one can define the extension $G_{c}$ of $G$ as the new game obtained by adding $c$ to $G$. Such a communication game proceeds as follows. In period 0 , after having received the output $m_{i}^{0}$, player $i$ is privately informed about his type $t_{i} \in T_{i}$, where $t=\left(t_{i}\right)_{i \in N}$ is distributed according to $p$. Then, at the beginning of each period $k \in\{1, \ldots, K\}$ he sends a confidential input $\left(r_{i}^{k}, s_{i}^{k}\right) \in R_{i}\left(t_{i}\right) \times S_{i}$ to the communication system. At the end of each period $k \in\{1, \ldots, K\}$, he receives a confidential output $m_{i}^{k} \in M_{i}$ from the communication system. Finally, after the last communication period (in period $K+1$, which corresponds to the action phase) he chooses an action $a_{i} \in A_{i}$ and is rewarded according to his utility function $u_{i}$.

A behavioral strategy for player $i$ in $G_{c}$ is a tuple $\left(\left(\sigma_{i}^{k}\right)_{k=1, \ldots, K}, \delta_{i}\right)$ where for all $k \in\{1, \ldots, K\}$,

$$
\sigma_{i}^{k}: M_{i}^{k} \times \mathcal{R}_{i}^{k-1} \times S_{i}^{k-1} \times T_{i} \rightarrow \Delta\left(\mathcal{R}_{i} \times S_{i}\right)
$$

is player $i$ 's communication strategy in period $k$ satisfying $\sigma_{i}^{k}\left(r_{i}^{k}, s_{i}^{k} \mid \cdot, t_{i}\right)=0$ 
whenever $r_{i}^{k} \notin R_{i}\left(t_{i}\right)$, and

$$
\delta_{i}: M_{i}^{K+1} \times \mathcal{R}_{i}^{K} \times S_{i}^{K} \times T_{i} \rightarrow \Delta\left(A_{i}\right),
$$

is player $i$ 's strategy in the action phase. A profile of behavioral strategies is denoted by $(\sigma, \delta)=\left(\sigma_{i}, \delta_{i}\right)_{i \in N}$, where $\sigma_{i}=\left(\sigma_{i}^{k}\right)_{k=1, \ldots, K}$. Such a strategy profile in $G_{c}$ generates an outcome $\mu: T \rightarrow \Delta(A)$ and an expected payoff $\sum_{t \in T} p(t) \sum_{a \in A} \mu(a \mid$ t) $u_{i}(a, t)$ for each player $i .{ }^{9}$ As usual, a (Bayesian) Nash equilibrium of the communication game $G_{c}$ is a strategy profile $(\sigma, \delta)$ such that no player can strictly increase his expected payoff by unilaterally deviating from his strategy. The outcome generated by a Nash equilibrium of $G_{c}$ is called an equilibrium outcome of $G_{c}{ }^{10}$

Definition 1 A certification equilibrium of $G$ is a Nash equilibrium of the extended game $G_{c}$ obtained by adding a communication system $c$ to $G .^{11}$

It can be shown ${ }^{12}$ that the set of all certification equilibrium outcomes, denoted by $\mathcal{E} \subseteq[\Delta(A)]^{T}$, obtained when considering all possible communication systems (in particular, all possible reporting correspondences), coincides with the set of Nash equilibrium outcomes of the extended games obtained by adding a one-period communication system $(K=1)$ without initial output $\left(\nu^{0}\right.$ is degenerate), without typeindependent input ( $S$ is a singleton), satisfying $M=A, R_{i}\left(t_{i}\right)=\left\{t_{i}\right\}$ for all $i \in N$ and $t_{i} \in T_{i}$, and in which every player follows the recommendation of the mediator. That is, a certification equilibrium outcome is simply characterized by a recommendation $\mu: T \rightarrow \Delta(A)$ satisfying

$\sum_{t_{-i} \in T_{-i}} p\left(t_{-i} \mid t_{i}\right) \sum_{a \in A} \mu(a \mid t) u_{i}(a ; t) \geq \sum_{t_{-i} \in T_{-i}} p\left(t_{-i} \mid t_{i}\right) \sum_{a \in A} \mu(a \mid t) u_{i}\left(a_{-i}, d_{i}\left(a_{i}\right) ; t\right)$

for all $i \in N, t_{i} \in T_{i}$, and $d_{i}: A_{i} \rightarrow A_{i}$. The intuition of this equivalent characterization is very simple. Starting with any certification equilibrium, the mediator first simulates the sequence of signals and reports (inputs) that would have been sent by the players and the sequence of messages (outputs) that would have been received by the players given the type profile under the original equilibrium. Then, he computes the actions that would have been chosen by the players as a function of the type profile and the sequence of inputs and outputs. Finally, he privately recommends

\footnotetext{
${ }^{9}$ That is, if for all $(m, r, s) \in M^{K} \times \mathcal{R}^{K} \times S^{K}$ we denote by $h\left(m, r, s \mid m^{0}, t\right)$ the probability distribution over $M^{K} \times \mathcal{R}^{K} \times S^{K}$ generated by $(\sigma, \delta)$ in $G_{c}$ given $m^{0} \in M$ and $t \in T$, then $\mu(a \mid t)=\sum_{m^{0} \in M} \nu^{0}\left(m^{0}\right) \sum_{(m, r, s) \in M^{K} \times \mathcal{R}^{K} \times S^{K}} h\left(m, r, s \mid m^{0}, t\right) \delta\left(a \mid m^{0}, m, r, s, t\right)$.

${ }^{10}$ We consider equilibrium outcomes rather than equilibrium strategies because the dimension of strategy sets depends on the underlying communication system. By contrast, equilibrium outcomes are always in $[\Delta(A)]^{T}$.

${ }^{11}$ We use the term "certification equilibrium" to point out the link with a communication equilibrium, which is defined as a certification equilibrium except that the communication systems used to define a communication equilibrium do not allow players to certify their information through type-dependent sets of available inputs (see Definition 2).

${ }^{12}$ The formal proof is a simplified version of the Proof of Theorem 1.
} 
each player to choose the associated action. Clearly, if a player has an incentive to deviate from the recommendation of the mediator, then the strategy profile of the original communication game was not an equilibrium.

The previous observation can be interpreted as a form of "revelation principle": any certification equilibrium is outcome equivalent to a "truthful certification equilibrium". However, the set of "truthful certification equilibria" generated in this way is much too large for the result to be interesting, and is not appropriate for most applications. Indeed, players may have the right to remain silent or to present only vague arguments, whereas in some certification equilibria they are compelled to reveal their type to the mediator even if they have no incentive to do so. A simple illustration is provided in Example 1. On the other hand, in some environments players may have only limited ability to certify claims. Accordingly, when certifiability possibilities are given and only partial, it is not appropriate to consider a communication system with $R_{i}\left(t_{i}\right)=\left\{t_{i}\right\}$ for all $i \in N$ and $t_{i} \in T_{i}$ because what is certified with such a communication system might not be certifiable with the original set of available reports.

For those reasons we define certification equilibria that can be obtained only with a specified profile of available type-dependent inputs, i.e., with communication systems where the reporting correspondences $R=\left(R_{i}\right)_{i \in N}$ are given. Such communication systems are called $R$-communication systems. If the set of available inputs does not depend on players' types then the set of associated equilibria is, by definition, the set of communication equilibria.

Definition 2 An $R$-certification equilibrium of $G$ is a Nash equilibrium of the extended game $G_{c}$ obtained by adding an $R$-communication system $c$ to $G$. A communication equilibrium is an $R$-certification equilibrium where $R_{i}\left(t_{i}\right)=R_{i}\left(t_{i}^{\prime}\right)$ for all $t_{i}, t_{i}^{\prime} \in T_{i}$ and $i \in N$.

We denote by $\mathcal{E}(R)$ the set of $R$-certification equilibrium outcomes and by $\mathcal{E}_{0}$ the set of communication equilibrium outcomes. Clearly, we have $\mathcal{E}_{0} \subseteq \mathcal{E}(R) \subseteq \mathcal{E}$ for every profile of reporting correspondences $R$, and all these sets are convex (thanks to the preliminary lottery $\nu^{0}$ ). As shown in the following example these inclusions may be strict.

Example 1 Consider a consumer whose endowments depend on two equally likely types, $t^{1}$ and $t^{2}$, which are private information to the consumer. There are two commodities. In state $t^{1}\left(t^{2}\right.$, resp.) the consumer's endowment is $(10,0)((0,10)$, resp.). A government can choose to deduct taxes of twenty per cent either on commodity 1 (action $a^{1}$ ) or on commodity 2 (action $a^{2}$ ). If each unit of commodity provides a utility of one to the consumer and to the government, this situation can be represented by the Bayesian game of Figure 1 on the next page. In this game it can be shown ${ }^{13}$ that the set of communication equilibrium outcomes and the set of $R$-certification

\footnotetext{
${ }^{13}$ See Section 3 for a general and explicit characterization.
} 
equilibrium outcomes coincide whenever player 1 can remain silent, i.e., whenever $\bigcap_{t \in T} R_{1}(t) \neq \emptyset$ : they are characterized by $\mu\left(a^{2} \mid t^{2}\right)=1-\mu\left(a^{1} \mid t^{1}\right)$. Hence, the only associated vector of expected payoffs is $(9,1)$. The set of all certification equilibrium outcomes is however strictly larger since it is the set of outcomes satisfying $\mu\left(a^{2} \mid t^{2}\right) \geq 1-\mu\left(a^{1} \mid t^{1}\right)$. In particular, the perfectly revealing recommendation induces such an equilibrium outcome with the vector of expected payoffs $(8,2)$.

\begin{tabular}{l|c|c|} 
& \multicolumn{1}{c}{$a^{1}$} & \multicolumn{1}{c}{$a^{2}$} \\
\cline { 3 - 3 }$t^{1}$ & $(8,2)$ & $(10,0)$ \\
\cline { 3 - 3 }$t^{2}$ & $(10,0)$ & $(8,2)$ \\
\hline
\end{tabular}

Figure 1: Bayesian Game of Example 1.

In the following section we introduce canonical communication systems and equilibria given some specified profile of reporting correspondences $R=\left(R_{i}\right)_{i \in N}$ in order to obtain a simple and equivalent characterization of the set of all $R$-certification equilibrium outcomes.

\section{Canonical Representations}

\subsection{Certifiability Configuration and Canonical Communication Sys- tems}

As noted earlier, the inputs in a communication system have no semantic content. In order to capture certification possibilities associated with a profile of reporting correspondences in a canonical way, we first introduce a framework where certifiable information is represented as events of the state space. Then, we prove a generalized version of the revelation principle for Bayesian games with type-dependent sets of available signals in order to characterize the set of all $R$-certification equilibrium outcomes in a tractable way. This is performed by defining appropriate canonical communication systems where the profile of reporting correspondences is written as a certifiability configuration.

A certifiability configuration is an $n$-tuple of collections of sets of types, $\mathcal{Y}=$ $\left(\mathcal{Y}_{i}\right)_{i \in N}$, where an element $y_{i} \in \mathcal{Y}_{i} \subseteq 2^{T_{i}} \backslash\{\emptyset\}$ is a certificate (certifiable event) concerning player $i$ 's type. For all $i \in N$ and $t_{i} \in T_{i}$ we assume that there exists $y_{i} \in \mathcal{Y}_{i}$ such that $t_{i} \in y_{i} .{ }^{14}$ The set of events that player $i$ of type $t_{i}$ is able to certify concerning his type is the set of certificates containing $t_{i}$ and is denoted by $Y_{i}\left(t_{i}\right) \equiv\left\{y_{i} \in \mathcal{Y}_{i}: t_{i} \in y_{i}\right\}$. Hence, a certifiability configuration $\mathcal{Y}=\left(\mathcal{Y}_{i}\right)_{i \in N}$ can equivalently be viewed as a profile of reporting correspondences $R=Y=\left(Y_{i}\right)_{i \in N}$. The closure of a certifiability configuration $Y$ is the certifiability configuration $\bar{Y}=$

\footnotetext{
${ }^{14}$ The set $\mathcal{Y}_{i}$ is not assumed to be closed under intersection, union or complementation, even if the closure under intersection often seems natural as will be discussed later.
} 
$\left(\bar{Y}_{i}\right)_{i \in N}$ where for all $i \in N$ and $t_{i} \in T_{i}, \bar{Y}_{i}\left(t_{i}\right)$ is the element of $\overline{\mathcal{Y}}_{i}$ containing $t_{i}$, and $\overline{\mathcal{Y}}_{i}$ is the smallest set containing $\mathcal{Y}_{i}$ which is closed under intersection. Define the smallest event concerning player $i$ 's type as Mini $Y_{i}\left(t_{i}\right) \equiv \bigcap_{y_{i} \in Y_{i}\left(t_{i}\right)} y_{i}$ and let Mini $Y(t)=\left(\operatorname{Mini} Y_{i}\left(t_{i}\right)\right)_{i \in N}$.

Let $R=\left(R_{i}\right)_{i \in N}$ be an arbitrary profile of reporting correspondences. With any such profile we can associate a unique certifiability configuration $Y^{R}=\left(Y_{i}^{R}\right)_{i \in N}$, where $Y_{i}^{R}\left(t_{i}\right) \equiv\left\{R_{i}^{-1}\left(r_{i}\right): r_{i} \in R_{i}\left(t_{i}\right)\right\}$ for all $t_{i} \in T_{i}, i \in N$, and $R_{i}^{-1}\left(r_{i}\right) \equiv$ $\left\{t_{i} \in T_{i}: r_{i} \in R_{i}\left(t_{i}\right)\right\}$ is the set of types of player $i$ who can send the report $r_{i}$. Hence, $\mathcal{Y}_{i}^{R} \equiv\left\{Y_{i}^{R}\left(t_{i}\right): t_{i} \in T_{i}\right\}=\left\{R_{i}^{-1}\left(r_{i}\right): r_{i} \in \mathcal{R}_{i}\right\}$ for all $i \in N$. It is worth mentioning that many different profiles of reporting correspondences can generate the same certifiability configuration.

Given a certifiability configuration $Y$ and its closure $\bar{Y}$, we define a canonical $\bar{Y}$-communication system as a $\bar{Y}$-communication system such that $S=T, M=A$, $K=1$, and $\nu^{0}$ is degenerate. Hence, in a canonical $\bar{Y}$-communication system there is no initial output, there is only one communication period, a report of each player $i \in N$ of type $t_{i} \in T_{i}$ is a certificate concerning his type, $y_{i} \in \bar{Y}_{i}\left(t_{i}\right)$, a cheap talk signal is a claim about his type, $s_{i} \in T_{i}$, and messages sent by the communication system are (recommended) actions.

\subsection{Canonical Certification Equilibria}

Definition 3 A canonical $\bar{Y}$-certification equilibrium of $G$ is a Nash equilibrium of the extended game $G_{c}$ obtained by adding a canonical $\bar{Y}$-communication system $c$ to $G$, and in which every player certifies the smallest event concerning his type, truthfully reveals his type, and follows the recommendation of the mediator.

In other words, in a canonical $\bar{Y}$-certification equilibrium each type $t_{i} \in T_{i}$ of every player $i \in N$ sends the report Mini $Y_{i}\left(t_{i}\right)$, sends the cheap talk signal $t_{i}$, and plays the action recommended by the mediator. Hence, such an equilibrium outcome is simply characterized by a recommendation (transition probability) $\nu^{*}: \overline{\mathcal{Y}} \times T \rightarrow$ $\Delta(A)$ satisfying

$$
\begin{aligned}
& \sum_{t_{-i} \in T_{-i}} p\left(t_{-i} \mid t_{i}\right) \sum_{a \in A} \nu^{*}(a \mid \operatorname{Mini} Y(t), t) u_{i}(a ; t) \geq \\
& \sum_{t_{-i} \in T_{-i}} p\left(t_{-i} \mid t_{i}\right) \sum_{a \in A} \nu^{*}\left(a \mid\left(\operatorname{Mini} Y_{-i}\left(t_{-i}\right), y_{i}\right),\left(t_{-i}, t_{i}^{\prime}\right)\right) u_{i}\left(a_{-i}, d_{i}\left(a_{i}\right) ; t\right),
\end{aligned}
$$

for all $i \in N, t_{i}, t_{i}^{\prime} \in T_{i}, y_{i} \in \bar{Y}_{i}\left(t_{i}\right)$, and $d_{i}: A_{i} \rightarrow A_{i}$. The set of canonical $\bar{Y}$-certification equilibrium outcomes is denoted by $\mathcal{E}^{*}(\bar{Y})$. According to the following theorem, for any profile of reporting correspondences $R=\left(R_{i}\right)_{i \in N}$, the set $\mathcal{E}^{*}\left(\bar{Y}^{R}\right)$, where $\bar{Y}^{R}$ is the closure of the certifiability configuration generated by $R$, exactly coincides with the set of all Nash equilibrium outcomes achievable through all $R$-communication systems. The intuition of this result is similar to the revelation principle for Bayesian games with non-certifiable information, except 
that, in the latter case, without any specific assumption, communication equilibria which use several communication periods can be equivalently achieved as one-stage canonical communication equilibria (see Forges, 1990). Here, we have to take the closure $\bar{Y}^{R}$ of the certifiability configuration $Y^{R}$ generated by the reporting correspondences $R$ to ensure that every information which can be certified by sending different reports at different periods in the original equilibrium can also be certified in the one-period canonical communication system. Deneckere and Severinov (2001) already recognized the crucial role of multiple reports in extending the revelation principle to principal-agent problems with partially verifiable types. Given a basic state space, they construct a large set of messages, which typically capture multiple claims about the agent's private information. Assuming the existence of a state independent "worst outcome", they show that any implementable social choice function, defined on the large set of messages, is truthfully implementable. Theorem 1 below differs from this result in several respects. First, it applies to any $n$-person Bayesian game, without any requirement of possible "worst outcomes". Furthermore, in an $R$-certification equilibrium, the range of the reporting correspondences, the input sets and the output sets have no relationship with the fundamentals of the game. We thus derive appropriate direct mechanisms before exhibiting truthful equilibria.

Theorem 1 The set of $R$-certification equilibrium outcomes coincides with the set of canonical $\bar{Y}^{R}$-certification equilibrium outcomes. That is, $\mathcal{E}(R)=\mathcal{E}^{*}\left(\bar{Y}^{R}\right)$ for all profiles of reporting correspondences $R$.

The following example illustrates the canonical representation. A similar, but not quite identical (see Subsection 3.3), example was used by Green and Laffont (1986) to show the possible failure of the revelation principle and by Deneckere and Severinov (2001) to show how restore it. The example also shows that communication equilibria can differ from certification equilibria even if we consider certifiability configurations $Y^{R}$ allowing players to remain silent, i.e., such that $T_{i} \in Y_{i}^{R}\left(t_{i}\right)$ for all $t_{i} \in T_{i}$ and $i \in N .^{15}$

Example 2 Consider the game of Figure 2 on the following page, where $N=\{1,2\}$, $T_{2}$ and $A_{1}$ are singleton, $T_{1}=\left\{t^{1}, t^{2}, t^{3}\right\}, A_{2}=\left\{a^{1}, a^{2}\right\}$, and consider the following reporting correspondence: $R\left(t^{1}\right)=\left\{r, r^{\prime}\right\}$ and $R\left(t^{2}\right)=R\left(t^{3}\right)=\left\{r, r^{\prime}, r^{\prime \prime}\right\}$. A naive application of the standard revelation principle in this game leads to the conclusion that the complete information outcome $\left(a^{1}\left|t^{1}, a^{2}\right| t^{2}, a^{2} \mid t^{3}\right)$ is not implementable since if each type sends a different report to the mediator, then the sender of type $t^{1}$ deviates by sending the same report as type $t^{2}$ or $t^{3}$. Consider on the contrary the canonical representation presented before. The certifiability configuration generated by $R$ is $\mathcal{Y}^{R}=\left\{\left\{t^{2}, t^{3}\right\}, T\right\}$ (the report $r^{\prime \prime}$ allows to exclude the occurrence of state $\left.t^{1}\right)$, so $\bar{Y}^{R}=Y^{R}$, Mini $Y^{R}\left(t^{1}\right)=T$ and $\operatorname{Mini} Y^{R}\left(t^{2}\right)=\operatorname{Mini} Y^{R}\left(t^{3}\right)=\left\{t^{2}, t^{3}\right\}$. The

\footnotetext{
${ }^{15}$ Note that this condition is equivalent to $\bigcap_{t_{i} \in T_{i}} R_{i}\left(t_{i}\right) \neq \emptyset$ for all $i \in N$. In other words, each player can send an uninformative report (i.e., a report which is available whatever his type).
} 
complete information outcome can be truthfully implemented with the recommendation $\nu^{*}: \mathcal{Y}^{R} \times T \rightarrow \Delta(A)$ satisfying $\nu^{*}\left(a^{2} \mid\left(\left\{t^{2}, t^{3}\right\}, t^{2}\right)\right)=\nu^{*}\left(a^{2} \mid\left(\left\{t^{2}, t^{3}\right\}, t^{3}\right)\right)=1$ and $\nu^{*}\left(a^{1} \mid(y, t)\right)=1$ for all other inputs $(y, t) \in \mathcal{Y}^{R} \times T$. Of course, this outcome is not a communication equilibrium outcome since type $t^{1}$ will claim that his type is $t^{2}$ or $t^{3}$.

\begin{tabular}{l|c|c|} 
& \multicolumn{1}{c}{$a^{1}$} & \multicolumn{1}{c}{$a^{2}$} \\
\cline { 2 - 3 }$t^{1}$ & $(0,1)$ & $(1,0)$ \\
\cline { 2 - 3 }$t^{2}$ & $(0,0)$ & $(1,1)$ \\
\cline { 2 - 3 }$t^{3}$ & $(0,0)$ & $(1,1)$ \\
\cline { 2 - 3 } & &
\end{tabular}

Figure 2: Bayesian Game of Example 2.

\subsection{One-Period Communication Systems}

In this subsection we give a sufficient condition on the profile of reporting correspondences $R$ such that the set of all Nash equilibrium outcomes that can be achieved with all one-period $R$-communication systems coincides with the set of $R$ certification equilibrium outcomes. The motivation for the restriction to Bayesian games extended with only one-period communication systems is that in some applications one may be interested by the set of equilibria that can be achieved when players are restricted to present only one or few arguments, as it is the case, e.g., in Glazer and Rubinstein's (2001) analysis of debates.

Another interesting example is the configuration examined by Alger and Renault (2002). There, the informed player can be of two different payoff-relevant types, $t^{1}$ and $t^{2}$, and in addition he can be honest or (possibly) dishonest. The honest player can only reveal his true payoff-relevant type, whereas the dishonest player can also lie. Denote by $t_{h}^{l}\left(t_{d}^{l}\right.$, resp.) the honest player (dishonest player, resp.) whose payoffrelevant type is $t^{l}$, for $l=1,2$. The reporting correspondence of the player is thus characterized by $R\left(t_{h}^{1}\right)=\left\{t^{1}\right\}, R\left(t_{h}^{2}\right)=\left\{t^{2}\right\}$, and $R\left(t_{d}^{1}\right)=R\left(t_{d}^{2}\right)=\left\{t^{1}, t^{2}\right\}$. This correspondence generates the certifiability configuration $\mathcal{Y}^{R}=\left\{\left\{t_{h}^{1}, t_{d}^{1}, t_{d}^{2}\right\},\left\{t_{h}^{2}, t_{d}^{1}, t_{d}^{2}\right\}\right\}$, and its closure is $\overline{\mathcal{Y}}^{R}=\left\{\left\{t_{h}^{1}, t_{d}^{1}, t_{d}^{2}\right\},\left\{t_{h}^{2}, t_{d}^{1}, t_{d}^{2}\right\},\left\{t_{d}^{1}, t_{d}^{2}\right\}\right\}$. Consider now the game of Figure 3 on the next page with a flat prior probability distribution. It is easy to see that there is an $R$-certification equilibrium generating the outcome $\mu\left(a^{1} \mid t_{d}^{1}\right)=$ $\mu\left(a^{1} \mid t_{d}^{2}\right)=\mu\left(a^{2} \mid t_{h}^{1}\right)=\mu\left(a^{3} \mid t_{h}^{2}\right)=1$. However, this equilibrium outcome cannot be achieved with any one-period $R$-communication system since one of the honest type will always imitate the input used by one of the dishonest type. As first pointed out by Deneckere and Severinov (2001), once multiple communication periods are allowed, a dishonest type can prove to be dishonest by sending two "contradicting" reports $\left(t^{1}\right.$ and $\left.t^{2}\right)$. This possibility is implicitly introduced by taking the closure of the original certifiability configuration, but is perhaps not satisfactory given the psychological considerations that motivate the example. In particular, following Al- 
ger and Renault's (2002) terminology, the "second-order honesty" configuration in which an honest player can neither imitate a dishonest player by lying about his payoff-relevant type nor by lying about his ethics becomes equivalent to the previous "first-order honesty" configuration in which an honest player is only required to tell the truth concerning his payoff-relevant type. ${ }^{16}$

\begin{tabular}{c|c|c|c|}
\multicolumn{1}{c}{} & \multicolumn{1}{c}{$a^{1}$} & \multicolumn{1}{c}{$a^{2}$} & $a^{3}$ \\
\cline { 2 - 4 }$t_{d}^{1}$ & $(2,1)$ & $(0,2)$ & $(1,-2)$ \\
\cline { 2 - 4 }$t_{h}^{1}$ & $(2,1)$ & $(0,2)$ & $(1,-2)$ \\
\cline { 2 - 4 }$t_{d}^{2}$ & $(2,1)$ & $(1,-2)$ & $(0,2)$ \\
\cline { 2 - 4 }$t_{h}^{2}$ & $(2,1)$ & $(1,-2)$ & $(0,2)$ \\
\cline { 2 - 4 } & &
\end{tabular}

Figure 3: One-Period vs Multiple-Period Certification Equilibria.

In the following lines we show that if each player is able to certify the intersection of all certifiable events concerning his type, then considering multiple periods or only single period communication systems is equivalent. Otherwise, as in the previous example, we are not able to provide a simple representation of the set of one-period certification equilibria since different inputs should be used to achieve different possible outcomes, and an initial lottery is thus necessary to ensure the convexity of the set of equilibrium outcomes.

Definition 4 A certifiability configuration $Y=\left(Y_{i}\right)_{i \in N}$, or an associated profile of reporting correspondences $R$ such that $Y^{R}=Y$, satisfies the Minimal Closure Condition (MCC) if Mini $Y_{i}\left(t_{i}\right) \in Y_{i}\left(t_{i}\right)$ for all $i \in N$ and $t_{i} \in T_{i}$.

Obviously, a sufficient but not necessary condition for MCC to be satisfied is that each collection of events $\mathcal{Y}_{i}$ is closed under intersection, i.e., $Y=\bar{Y}$. Another sufficient condition for a certifiability configuration to satisfy MCC is that it is generated by a profile of reporting correspondences satisfying Green and Laffont's (1986) Nested Range Condition (NRC). More precisely, a profile of reporting correspondences $R$ such that $t_{i} \in R_{i}\left(t_{i}\right) \subseteq T_{i}$ for all $i \in N$ and $t_{i} \in T_{i}$ satisfies NRC if for all $i \in N$ and $t_{i}, t_{i}^{\prime} \in T_{i}$ we have $t_{i}^{\prime} \in R_{i}\left(t_{i}\right) \Rightarrow R_{i}\left(t_{i}^{\prime}\right) \subseteq R_{i}\left(t_{i}\right)$. It is not difficult to prove that under NRC the generated certifiability configuration satisfies MCC. However, the converse is not true. Indeed, consider a reporting correspondence as in Example 2: $T=\left\{t^{1}, t^{2}, t^{3}\right\}, R\left(t^{1}\right)=\left\{t^{1}, t^{2}\right\}, R\left(t^{2}\right)=R\left(t^{3}\right)=T$. NRC is not satisfied since $t^{2} \in R\left(t^{1}\right)$ but $R\left(t^{2}\right) \nsubseteq R\left(t^{1}\right)$. However, MCC is satisfied since the

\footnotetext{
${ }^{16} \mathrm{It}$ is important to notice the difference between this example and Example 2. In Example 2 the complete information outcome cannot be achieved by requiring that every player sends a different type-dependent input but it can be achieved with the original reporting correspondence as an equilibrium in which the mediator cannot distinguish type $t^{2}$ from type $t^{3}$. On the contrary, the outcome considered in the previous example cannot be obtained as an equilibrium with the original reporting correspondence.
} 
generated set of certifiable events, $\mathcal{Y}^{R}=\left\{\left\{t^{2}, t^{3}\right\}, T\right\}$, is closed under intersection. ${ }^{17}$

Theorem 2 If $R$ satisfies the minimal closure condition, then the set of one-period $R$-certification equilibrium outcomes coincides with the set of $R$-certification equilibrium outcomes.

An immediate corollary of Theorems 1 and 2 is that under MCC the set of all one-period $R$-certification equilibrium outcomes exactly coincides with the set of canonical $\bar{Y}^{R}$-certification equilibrium outcomes. It is also worth mentioning that under MCC a canonical $Y^{R}$-certification equilibrium is well defined and that $\mathcal{E}^{*}\left(\bar{Y}^{R}\right)=\mathcal{E}^{*}\left(Y^{R}\right)$ even if $\bar{Y}^{R} \neq Y^{R}$.

\subsection{An Alternative Representation}

In this subsection, following the approach of Forges et al. (2002), ${ }^{18}$ we propose an alternative representation theorem for Bayesian games with certifiable information by constructing, from any given $R$-communication system, an $R^{*}$-communication system in which the set of available inputs of each type $t_{i}$ of every player $i$ is restricted to a subset $R_{i}^{*}\left(t_{i}\right)$ of his set of types (i.e., $R_{i}^{*}\left(t_{i}\right) \subseteq T_{i}$ for all $t_{i} \in T_{i}$ and $i \in N$ ). Such a communication system can be (uniquely) defined for any $R$-communication system, and the associated set of equilibrium outcomes contains all $R$-certification equilibrium outcomes. However, in general, this set does not coincide with the set of $R$-certification equilibrium outcomes because it may contain more outcomes than can actually be achieved with $R$-communication systems. Nevertheless, natural sufficient conditions are provided for the equivalence to hold.

More precisely, given any profile of reporting correspondences $R$, let $R_{i}^{*}\left(t_{i}\right) \equiv$ $\left\{s_{i} \in T_{i}: \operatorname{Mini} Y_{i}^{R}\left(s_{i}\right) \in \bar{Y}_{i}^{R}\left(t_{i}\right)\right\}$ for all $t_{i} \in T_{i}$ and $i \in N$. That is, in an $R^{*}$ communication system the set of all type-dependent inputs that the mediator can receive from each player is a claim concerning his type, where it is implicitly assumed that when some type $t_{i}$ is reported by player $i$ he also sends the associated certificate Mini $Y_{i}^{R}\left(t_{i}\right)$. It is not difficult to check that the profile of correspondences $R^{*}$ generates the certifiability configuration $\widetilde{\mathcal{Y}}^{R}=\left(\widetilde{\mathcal{Y}}_{i}^{R}\right)_{i \in N}$, where for all $i \in N$, $\widetilde{\mathcal{Y}}_{i}^{R} \equiv\left\{\operatorname{Mini} Y_{i}^{R}\left(t_{i}\right): t_{i} \in T_{i}\right\} .{ }^{19}$ Hence, from Theorem 1 we know that the set of $R^{*}$-certification equilibrium outcomes coincides with the set of $\widetilde{Y}^{R}$-certification equilibrium outcomes. Moreover, since players have less possible deviations in a

\footnotetext{
${ }^{17}$ In Green and Laffont's (1986) original example, mentioned in the previous section, neither NRC nor MCC are satisfied, so that Theorem 2 does not apply.

${ }^{18}$ Forges et al. (2002) consider an exchange economy with type-dependent preferences and initial endowments. By relying on an appropriate version of the revelation principle, they focus on mechanisms in which every agent is just asked to report his type, with the understanding that he has to show the corresponding initial endowment.

${ }^{19}$ Of course, when certification possibilities are partial, this implies that players can still lie concerning their true type. For example, if Mini $Y_{i}^{R}\left(s_{i}\right) \in \widetilde{Y}_{i}^{R}\left(t_{i}\right)$ for $s_{i} \neq t_{i}$, then type $t_{i}$ can certify Mini $Y_{i}^{R}\left(s_{i}\right) \neq \operatorname{Mini} Y_{i}^{R}\left(t_{i}\right)$. This cannot happen, however, if all types can be fully certified, i.e., if $\left\{t_{i}\right\} \in \overline{\mathcal{Y}}_{i}^{R}$ for all $i \in N$ and $t_{i} \in T_{i}$.
} 
(canonical) $\tilde{Y}^{R}$-certification equilibrium than in a (canonical) $\bar{Y}^{R}$-certification equilibrium, the set of $R$-certification equilibrium outcomes is included in the set of $R^{*}$-certification equilibrium outcomes. The next theorem shows that we can even consider one-period $R^{*}$-certification equilibria without initial outputs and without cheap talk signals, where every player truthfully reveals his type and follows the recommendation of the mediator.

Theorem 3 Every $R$-certification equilibrium is outcome-equivalent to a one-period $R^{*}$-certification equilibrium in which the communication system has no initial output, $S$ is a singleton, $M=A$, and $R_{i}^{*}\left(t_{i}\right) \equiv\left\{s_{i} \in T_{i}: \operatorname{Mini} Y_{i}^{R}\left(s_{i}\right) \in \bar{Y}_{i}^{R}\left(t_{i}\right)\right\}$ for all $i \in N$ and $t_{i} \in T_{i}$, and in which strategies are truthful and obedient.

For example, the complete information outcome obtained in Example 2 can be truthfully implemented with this alternative representation, which gives $R^{*}\left(t^{1}\right)=$ $\left\{t^{1}\right\}$ and $R^{*}\left(t^{2}\right)=R^{*}\left(t^{3}\right)=\left\{t^{1}, t^{2}, t^{3}\right\}$. In this example the modification of the reporting correspondence $R$ is irrelevant since the closure of the generated certifiability configuration is not modified $\left(\bar{Y}^{R}=\widetilde{Y}^{R}\right)$. However, in general, the closure of the certifiability configuration generated by $R$ is different from the certifiability configuration generated by $R^{*}$, so the inclusion in Theorem 3 may be strict (see Example 1).

The equivalence is restored, for example, if the mediator is able to impose a penalty to any player whose report does not correspond to any equilibrium report, i.e., if for all $i \in N$ and $t_{-i} \in T_{-i}$ there exists $a_{-i} \in A_{-i}$ such that $u_{i}\left(a_{i}, a_{-i} ; t\right) \leq$ $u_{i}\left(a^{\prime} ; t\right)$ for all $a_{i} \in A_{i}, a^{\prime} \in A$ and $t_{i} \in T_{i}$. Recalling the comments in Subsection 3.2, $a_{-i}$ is a "worst outcome" in the sense of Deneckere and Severinov (2001). This assumption is for instance satisfied in the standard mechanism design framework with transferable utility, where there are $n-1$ agents (with no decision to make) and one uninformed player (the principal) who can make monetary transfers between agents. Alternatively, a mechanism designer or a mediator may be able to directly restrict the set of reporting choices of the individuals (albeit not being able to prevent them from lying), as it is the case when positive disclosures are mandatory. Under one of these conditions, an interesting corollary of Theorem 2 is that under MCC the set of all one-period $R$-certification equilibrium outcomes exactly coincides with the set of truthful and obedient one-period $R^{*}$-certification equilibrium outcomes. This characterization may be very useful in many applications since a truthful and obedient one-period $R^{*}$-certification equilibrium is simply characterized by an outcome function $\mu: T \rightarrow \Delta(A)$ satisfying

$$
\begin{aligned}
& \sum_{t_{-i} \in T_{-i}} p\left(t_{-i} \mid t_{i}\right) \sum_{a \in A} \mu(a \mid t) u_{i}(a ; t) \geq \\
& \sum_{t_{-i} \in T_{-i}} p\left(t_{-i} \mid t_{i}\right) \sum_{a \in A} \mu\left(a \mid t_{-i}, t_{i}^{\prime}\right) u_{i}\left(a_{-i}, d_{i}\left(a_{i}\right) ; t\right),
\end{aligned}
$$


for all $i \in N, t_{i} \in T_{i}, t_{i}^{\prime} \in R^{*}\left(t_{i}\right)$, and $d_{i}: A_{i} \rightarrow A_{i}$.

Finally, it is interesting to remark that the approach proposed here allows to make a direct link with Green and Laffont's (1986) framework. Indeed, it can be checked that a profile of reporting correspondences $R$ satisfies NRC if and only if $R=R^{*}$. As a consequence, if one of the conditions discussed in the previous paragraph is satisfied, then for any profile $R$ we can construct unambiguously, and without loss of generality, another profile $R^{*}$ satisfying NRC. Otherwise, in the general case, the canonical construction of the representation theorem 1 or 2 should be used.

\section{Concluding Remarks}

In this paper we have characterized in a tractable way the set of all Nash equilibrium outcomes that can be achieved in Bayesian games in which players have the ability to voluntarily certify and exchange their information through general communication systems. In particular, our framework and results encompass the representation theorem for communication equilibria, as well as existing versions of the revelation principle for principal-agent problems where the set of reports available to the agent is type-dependent.

Since we have considered general communication systems the question of how certification equilibrium outcomes can be implemented in an equilibrium by adding only unmediated communication systems to the original Bayesian game was not addressed in this paper and remains the topic of future research. In particular, it should be interesting to investigate whether certification equilibrium outcomes can be implemented with direct communication systems by considering a sufficient number of players (as, e.g., in Bárány, 1992, Ben-Porath, 2003, Forges, 1990, and Gerardi, 2003), by allowing codified messages and bounded computational abilities (as in Urbano and Vila, 2002), or by considering the correlated equilibrium instead of the Nash equilibrium as a solution concept (as in Forges, 1988). It should also be helpful to provide a geometric characterization of the set of Nash equilibrium outcomes achievable with direct communication and certifiable information in twoplayer games with incomplete information on one side, as is provided by Aumann and Hart (2003) for cheap talk communication. There, the set of communication equilibrium outcomes gives an upper bound for the set of Nash equilibrium outcomes achievable with unmediated communication systems when information is not certifiable. The set of certification equilibrium outcomes characterized in this paper gives exactly the analog of this upper bound in direct communication games with partially verifiable types.

\section{Appendix}

To prove the theorems we introduce some lemmas and some additional notations. Denote by $\mathcal{E}(R \mid K=1)$ the set of one-period $R$-certification equilibrium outcomes, 
and denote by $\mathcal{E}^{\#}\left(R^{*}\right)$ the set of one-period $R^{*}$-certification equilibrium outcomes in which the communication system has no initial output, $S$ is a singleton, $M=A$, and in which strategies are truthful and obedient. Let $Q^{R}=\left(Q^{R}\right)_{i \in N}$ be the profile of correspondences defined by $Q_{i}^{R}\left(t_{i}\right) \equiv\left\{q_{i} \in 2^{\mathcal{R}_{i}}: q_{i} \subseteq R_{i}\left(t_{i}\right)\right\}$ for all $t_{i} \in T_{i}$ and $i \in N$. Clearly, we have $Y^{Q^{R}}=\bar{Y}^{Q^{R}}=\bar{Y}^{R}$.

Lemma 1 If Mini $Y_{i}^{R}\left(t_{i}^{\prime}\right) \in \bar{Y}_{i}^{R}\left(t_{i}\right)$, then $R_{i}\left(t_{i}^{\prime}\right) \subseteq R_{i}\left(t_{i}\right)$.

Proof. We show that $R_{i}\left(t_{i}^{\prime}\right) \nsubseteq R_{i}\left(t_{i}\right) \Rightarrow \operatorname{Mini} Y_{i}^{R}\left(t_{i}^{\prime}\right) \notin \bar{Y}_{i}^{R}\left(t_{i}\right)$. Let $r_{i} \in R_{i}\left(t_{i}^{\prime}\right)$, $r_{i} \notin R_{i}\left(t_{i}\right)$. We have $r_{i} \in R_{i}\left(t_{i}^{\prime}\right) \Rightarrow R_{i}^{-1}\left(r_{i}\right) \in \bar{Y}_{i}^{R}\left(t_{i}^{\prime}\right) \Rightarrow \operatorname{Mini} Y_{i}^{R}\left(t_{i}^{\prime}\right) \subseteq R_{i}^{-1}\left(r_{i}\right)$, and $r_{i} \notin R_{i}\left(t_{i}\right) \Rightarrow t_{i} \notin R_{i}^{-1}\left(r_{i}\right)$. Thus, $t_{i} \notin \operatorname{Mini} Y_{i}^{R}\left(t_{i}^{\prime}\right)$, which implies that Mini $Y_{i}^{R}\left(t_{i}^{\prime}\right) \notin$ $\bar{Y}_{i}^{R}\left(t_{i}\right)$ since $t_{i} \in y_{i}$ for all $y_{i} \in \bar{Y}_{i}^{R}\left(t_{i}\right)$.

Lemma 2 For every profile of reporting correspondences $R, \mathcal{E}^{*}\left(\bar{Y}^{R}\right) \subseteq \mathcal{E}(R)$. If $R$ satisfies $M C C$, then $\mathcal{E}^{*}\left(\bar{Y}^{R}\right) \subseteq \mathcal{E}(R \mid K=1)$.

Proof. Let $\nu^{*}: \overline{\mathcal{Y}}^{R} \times T \rightarrow \Delta(A)$ be any canonical $\bar{Y}^{R}$-certification equilibrium. We construct an outcome-equivalent $R$-certification equilibrium as follows. Let $c$ be an $R$-communication system satisfying $M=A, S=T, K>\left|R_{i}\left(t_{i}\right)\right|$ for all $i \in N$ and $t_{i} \in T_{i}, \nu^{k}$ is degenerate for $k=0,1, \ldots, K-1$. In addition, $\nu^{K}$ only depends on the sequence of reporting profiles $r=\left(r^{1}, \ldots, r^{K}\right) \in \mathcal{R}^{K}$ and on the cheap talk signals sent in the last communication period $(\operatorname{period} K), s^{K}=\left(s_{1}^{K}, \ldots, s_{n}^{K}\right) \in T$. More precisely, let

$$
\nu^{K}(m, r, s)=\nu^{*}\left(\left[\bigcap_{k \in\{1, \ldots, K\}} R_{i}^{-1}\left(r_{i}^{k}\right)\right]_{i \in N}, s^{K}\right)
$$

for all $(m, r, s) \in M^{K} \times \mathcal{R}^{K} \times T^{K}$. Since $\overline{\mathcal{Y}}_{i}^{R}=\left\{\bigcap_{k \in\{1, \ldots, K\}} R_{i}^{-1}\left(r_{i}^{k}\right): r_{i} \in \mathcal{R}_{i}^{K}\right\}$ and $\bigcap_{r_{i} \in R_{i}\left(t_{i}\right)} R_{i}^{-1}\left(r_{i}\right)=\operatorname{Mini} Y_{i}^{R}\left(t_{i}\right)$ for all $i \in N$, the strategy which consists for each type $t_{i}$ of every player $i$ in sending every report in $R_{i}\left(t_{i}\right)$ during the communication phase, revealing his true type in the last communication period and following the recommendation of the mediator is, by the definition of the original canonical $\bar{Y}^{R}$ certification equilibrium and the construction of $c$, a Nash equilibrium of $G_{c}$. This equilibrium is clearly outcome-equivalent to $\nu^{*}$. Similarly, to prove the second part of the lemma let $\nu(m, r, s)=\nu^{*}\left(\left[R_{i}^{-1}\left(r_{i}\right)\right]_{i \in N}, s\right)$ for all $(m, r, s) \in M \times \mathcal{R} \times T$ and remark that under MCC, for all $i \in N$ and $t_{i} \in T_{i}$, there exists $r_{i} \in R_{i}\left(t_{i}\right)$ such that $R_{i}^{-1}\left(r_{i}\right)=\operatorname{Mini} Y_{i}^{R}\left(t_{i}\right)$.

Lemma 3 For every profile of reporting correspondences $R, \mathcal{E}(R) \subseteq \mathcal{E}\left(Q^{R} \mid K=1\right)$.

Proof. Consider any Nash equilibrium of any communication game $G_{c}$ where $c$ is an $R$-communication system. We construct an outcome-equivalent one-period $Q^{-}$ certification equilibrium where $Q=Q^{R}, M=A$, the initial lottery is degenerate, the 
transition probability is $\pi:\left(\prod_{i \in N} 2^{\mathcal{R}_{i}}\right) \times T \rightarrow \Delta(A)$, each player $i$ of type $t_{i}$ follows the recommendation generated by $\pi$, sends the report $R_{i}\left(t_{i}\right) \in Q\left(t_{i}\right)$ and reveals his true type. That is, $\sigma_{i}\left(t_{i}\right)=\left(R_{i}\left(t_{i}\right), t_{i}\right)$ and $\delta_{i}\left(a_{i} \mid a_{i}, r_{i}, s_{i}, t_{i}\right)=1$ for all $t_{i} \in T_{i}$, $a_{i} \in A_{i},\left(r_{i}, s_{i}\right) \in Q_{i}\left(t_{i}\right) \times T_{i}$ and $i \in N$. If every player $i$ sends an input $\left(R_{i}\left(s_{i}\right), s_{i}\right)$ for some $s_{i} \in T_{i}$, then $\pi$ simulates the action profile played in the original equilibrium when the type profile is $s=\left(s_{1}, \ldots, s_{n}\right) \in T$. Clearly, this constructed mechanism generates the original equilibrium outcome. To verify that it is incentive compatible we must verify that for every player $i$, no type $t_{i}$ has an incentive to deviate from $\left(R_{i}\left(t_{i}\right), t_{i}\right)$ to $\left(q_{i}, s_{i}\right) \neq\left(R_{i}\left(t_{i}\right), t_{i}\right)$ for all $\left(q_{i}, s_{i}\right) \in Q_{i}\left(t_{i}\right) \times T_{i}$. If $\left(q_{i}, s_{i}\right)=\left(R_{i}\left(s_{i}\right), s_{i}\right)$ (unobservable deviation), then $R_{i}\left(s_{i}\right) \subseteq R_{i}\left(t_{i}\right)$ (because $R_{i}\left(s_{i}\right) \in Q_{i}\left(t_{i}\right) \Rightarrow R_{i}\left(s_{i}\right) \subseteq$ $R_{i}\left(t_{i}\right)$ ), which means that type $t_{i}$ already had the possibility to imitate type $s_{i}$ 's communication strategy under the original equilibrium. If $\left(q_{i}, s_{i}\right) \neq\left(R_{i}\left(s_{i}\right), s_{i}\right)$ (observable deviation), then $\pi$ simulates the outcome generated by a deviation of player $i$ to, e.g., an unconditional sequence of $K$ reports of any single report in $q_{i}$ and $K$ cheap talk signals in $S_{i}$ under the original equilibrium. This deviation was already available to type $t_{i}$ since $q_{i} \in Q_{i}\left(t_{i}\right) \Rightarrow q_{i} \subseteq R_{i}\left(t_{i}\right)$.

Lemma 4 For every profile of reporting correspondences $R, \mathcal{E}(R \mid K=1) \subseteq$ $\mathcal{E}^{*}\left(\bar{Y}^{R}\right)$.

Proof. The proof is similar to the proof of Lemma 3. Consider any Nash equilibrium of any communication game $G_{c}$ where $c$ is a one-period $R$-communication system. We construct an outcome-equivalent canonical $\bar{Y}$-certification equilibrium $\nu^{*}: \overline{\mathcal{Y}} \times T \rightarrow \Delta(A)$, where $\bar{Y}=\bar{Y}^{R}$, as follows. If every player $i$ sends an input (Mini $\left.Y_{i}\left(s_{i}\right), s_{i}\right)$ for some $s_{i} \in T_{i}$, then $\nu^{*}$ simulates the action profile played in the original equilibrium when the type profile is $s \in T$. If some player $i$ sends an input $\left(y_{i}, s_{i}\right) \neq\left(\operatorname{Mini} Y_{i}\left(s_{i}\right), s_{i}\right)$, then $\nu^{*}$ simulates the outcome generated by player $i$ 's deviation to some report $r_{i}$ such that $y_{i} \subseteq R_{i}^{-1}\left(r_{i}\right)$ and some cheap talk signal in $S_{i}$ under the original equilibrium. This deviation was already available to type $t_{i}$ since $y_{i} \in \bar{Y}_{i}\left(t_{i}\right) \Rightarrow t_{i} \in y_{i} \subseteq R_{i}^{-1}\left(r_{i}\right) \Rightarrow r_{i} \in R_{i}\left(t_{i}\right)$. It remains to show that type $t_{i}$ has no incentive to send an input (Mini $\left.Y_{i}\left(s_{i}\right), s_{i}\right)$ for $s_{i} \neq t_{i}$. This is obtained by Lemma 1 since Mini $Y_{i}\left(s_{i}\right) \in Y_{i}\left(t_{i}\right)$, so $R_{i}\left(s_{i}\right) \subseteq R_{i}\left(t_{i}\right)$, which means that an equivalent deviation was already available under the original equilibrium.

Proof of Theorem 1. Lemma 4 gives $\mathcal{E}\left(Q^{R} \mid K=1\right) \subseteq \mathcal{E}^{*}\left(\bar{Y}^{Q^{R}}\right)=\mathcal{E}^{*}\left(\bar{Y}^{R}\right)$. Therefore, by Lemmas 2 and 3 we get $\mathcal{E}\left(Q^{R} \mid K=1\right) \subseteq \mathcal{E}^{*}\left(\bar{Y}^{R}\right) \subseteq \mathcal{E}(R) \subseteq \mathcal{E}\left(Q^{R} \mid\right.$ $K=1)$, so $\mathcal{E}^{*}\left(\bar{Y}^{R}\right)=\mathcal{E}(R)$.

Lemma 5 For every profile of reporting correspondences $R, \mathcal{E}^{\#}\left(R^{*}\right)=\mathcal{E}^{*}\left(\widetilde{Y}^{R}\right)$.

Proof. Clearly, we have $\mathcal{E}^{\#}\left(R^{*}\right) \subseteq \mathcal{E}\left(R^{*}\right)$. In addition, Theorem 1 gives $\mathcal{E}\left(R^{*}\right)=\mathcal{E}^{*}\left(\widetilde{Y}^{R}\right)$ because the profile of reporting correspondences $R^{*}$ generates the certifiability configuration $\widetilde{Y}^{R}$. Thus, we have to show that $\mathcal{E}^{*}\left(\widetilde{Y}^{R}\right) \subseteq \mathcal{E}^{\#}\left(R^{*}\right)$. Let 
$\nu^{*}: \widetilde{\mathcal{Y}}^{R} \times T \rightarrow \Delta(A)$ be any canonical $\widetilde{Y}^{R}$-certification equilibrium. We have to show that $\mu: T \rightarrow \Delta(A)$, where $\mu(a \mid t)=\nu^{*}\left(a \mid \operatorname{Mini} Y^{R}(t), t\right)$, induces a truthful and obedient one-period $R^{*}$-certification equilibrium outcome (an outcome in $\mathcal{E}^{\#}\left(R^{*}\right)$ ), i.e., that Equation (2) on page 14 is satisfied for all $t_{i}^{\prime} \in R^{*}\left(t_{i}\right)$ and $d_{i}: A_{i} \rightarrow A_{i}$. Since $t_{i}^{\prime} \in R^{*}\left(t_{i}\right) \Leftrightarrow \operatorname{Mini} Y_{i}^{R}\left(t_{i}^{\prime}\right) \in \widetilde{Y}_{i}^{R}\left(t_{i}\right)$, this condition is implied by the fact that $\nu^{*}$ is a $\widetilde{Y}^{R}$-certification equilibrium outcome (see Equation (1) on page 9 with $\left.Y=\bar{Y}=\widetilde{Y}^{R}\right)$.

Proof of Theorem 2. Let $R$ be a profile of reporting correspondences satisfying MCC. By Lemma 4 we have $\mathcal{E}(R \mid K=1) \subseteq \mathcal{E}^{*}\left(\bar{Y}^{R}\right)$, and by Lemma 2 we have $\mathcal{E}^{*}\left(\bar{Y}^{R}\right) \subseteq \mathcal{E}(R \mid K=1)$, so $\mathcal{E}^{*}\left(\bar{Y}^{R}\right)=\mathcal{E}(R \mid K=1)$. Thus, by Theorem 1 we get $\mathcal{E}(R)=\mathcal{E}(R \mid K=1)$.

Proof of Theorem 3. We have $\mathcal{E}(R)=\mathcal{E}^{*}\left(\bar{Y}^{R}\right)$ by Theorem $1, \mathcal{E}^{*}\left(\bar{Y}^{R}\right) \subseteq \mathcal{E}^{*}\left(\widetilde{Y}^{R}\right)$ because players have less possible deviations in a canonical $\widetilde{Y}^{R}$-certification equilibrium than in a canonical $\bar{Y}^{R}$-certification equilibrium, and $\mathcal{E}^{*}\left(\widetilde{Y}^{R}\right)=\mathcal{E}^{\#}\left(R^{*}\right)$ by Lemma 5. Consequently, $\mathcal{E}(R) \subseteq \mathcal{E}^{\#}\left(R^{*}\right)$.

\section{References}

Alger, I. AND C.-T. A. MA (2003): "Moral Hazard, Insurance, and some Collusion," Journal of Economic Behavior and Organization, 50, 225-247.

Alger, I. And R. Renault (2002): "Screening Ethics when Honest Agents Care about Fairness," mimeo.

Aumann, R. J. (1974): "Subjectivity and Correlation in Randomized Strategies," Journal of Mathematical Economics, 1, 67-96.

Aumann, R. J. And S. Hart (2003): "Long Cheap Talk," Econometrica, forthcoming.

Baliga, S. AND S. Morris (2002): "Co-ordination, Spillovers, and Cheap Talk," Journal of Economic Theory, 105, 450-468.

BÁrÁny, I. (1992): "Fair Distribution Protocols or How the Players Replace Fortune," Mathematics of Operations Research, 17, 327-340.

Ben-Porath, E. (2003): "Cheap Talk in Games with Incomplete Information," Journal of Economic Theory, 108, 45-71.

Bull, J. And J. Watson (2002): "Hard Evidence and Mechanism Design," mimeo, University of California, San Diego.

Che, Y.-K. And I. Gale (2000): "The Optimal Mechanism for Selling to a BudgetConstrained Buyer," Journal of Economic Theory, 92, 198-233.

Crawford, V. P. And J. Sobel (1982): "Strategic Information Transmission," Econometrica, 50, 1431-1451. 
D'Aspremont, C., S. Bhattacharya, and L.-A. Gérard-Varet (2000): "Bargaining and Sharing Innovative Knowledge," Review of Economic Studies, 67, $255-271$.

Deneckere, R. And S. Severinov (2001): "Mechanism Design and Communication Costs," mimeo, University of Wisconsin-Madison.

Farrell, J. And M. Rabin (1996): "Cheap Talk," Journal of Economic Perspectives, 10, 103-118.

Forges, F. (1986): "An Approach to Communication Equilibria," Econometrica, $54,1375-1385$.

(1988): "Can Sunspots Replace a Mediator?" Journal of Mathematical Economics, 17, 347-368.

_ (1990): "Universal Mechanisms," Econometrica, 58, 1341-1364.

Forges, F., J.-F. Mertens, and R. Vohra (2002): "The Ex Ante Incentive Compatible Core in the Absence of Wealth Effects," Econometrica, 70, 1865-1892.

Gerardi, D. (2003): "Unmediated Communication in Games with Complete and Incomplete Information," Journal of Economic Theory, forthcoming.

Glazer, J. And A. Rubinstein (2001): "Debates and Decisions: On a Rationale of Argumentation Rules," Games and Economic Behavior, 36, 158-173.

Green, J. R. And J.-J. LAfFont (1986): "Partially Verifiable Information and Mechanism Design," Review of Economic Studies, 53, 447-456.

Grossman, S. J. (1981): "The Informational Role of Warranties and Private Disclosure about Product Quality," Journal of Law and Economics, 24, 461-483.

Grossman, S. J. And O. D. Hart (1980): "Disclosure Laws and Takeover Bids," Journal of Finance, 35, 323-334.

Koessler, F. (2002): "Strategic Knowledge Sharing in Bayesian Games," mimeo, THEMA, Université de Cergy-Pontoise.

_ (2003): "Persuasion Games with Higher-Order Uncertainty," Journal of Economic Theory, forthcoming.

Krishna, V. And J. Morgan (2002): "The Art of Conversation," mimeo.

Lipman, B. L. And D. Seppi (1995): "Robust Inference in Communication Games with Partial Provability," Journal of Economic Theory, 66, 370-405.

Milgrom, P. (1981): "Good News and Bad News: Representation Theorems and Applications," Bell Journal of Economics, 12, 380-391.

Myerson, R. B. (1982): "Optimal Coordination Mechanisms in Generalized Principal-Agent Problems," Journal of Mathematical Economics, 10, 67-81. 
358

(1986): "Multistage Games with Communication," Econometrica, 54, 323358.

(1994): "Communication, Correlated Equilibria and Incentive Compatibility," in Handbook of Game Theory, ed. by R. J. Aumann and S. Hart, Elsevier Science B. V., vol. 2, chap. 24, 827-847.

Okuno-Fujiwara, A., M. Postlewaite, And K. Suzumura (1990): "Strategic Information Revelation," Review of Economic Studies, 57, 25-47.

Seidmann, D. J. And E. Winter (1997): "Strategic Information Transmission with Verifiable Messages," Econometrica, 65, 163-169.

Shin, H. S. (1994): "The Burden of Proof in a Game of Persuasion," Journal of Economic Theory, 64, 253-264.

_ (2003): "Disclosures and Asset Returns," Econometrica, 71, 105-133.

Urbano, A. And J. E. Vila (2002): "Computational Complexity and Communication: Coordination in Two-Player Games," Econometrica, 70, 1893-1927.

Verrecchia, R. E. (2001): "Essays on Disclosure," Journal of Accounting and Economics, 32, 97-180.

Wolinsky, A. (2003): "Information Transmission when the Sender's Preferences are Uncertain," Games and Economic Behavior, forthcoming. 